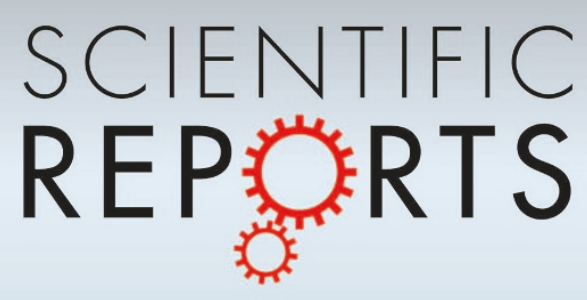

OPEN

SUBJECT AREAS:

SUPERCONDUCTING PROPERTIES AND

MATERIALS

METALS AND ALLOYS

ELECTRONIC PROPERTIES AND MATERIALS

STRUCTURAL GEOLOGY

Received

5 June 2013

Accepted

12 November 2013

Published

29 November 2013

Correspondence and requests for materials should be addressed to B.W. (bswang@issp. u-tokyo.ac.jp) or K.O. (ohgushi@issp.u-tokyo.

ac.jp)

\section{Superconductivity in anti-post-perovskite vanadium compounds}

\author{
Bosen Wang \& Kenya Ohgushi
}

Institute for Solid State Physics, University of Tokyo, Kashiwanoha, Kashiwa, Chiba 277-8581, Japan.

Superconductivity, which is a quantum state induced by spontaneous gauge symmetry breaking, frequently emerges in low-dimensional materials. Hence, low dimensionality has long been considered as necessary to achieve high superconducting transition temperatures $\left(T_{\mathrm{C}}\right)$. The recently discovered post-perovskite (ppv) $\mathrm{MgSiO}_{3}$, which constitutes the Earth's lowermost mantle (D" layer), has attracted significant research interest due to its importance in geoscience. The ppv structure has a peculiar two-dimensional character and is expected to be a good platform for superconductivity. However, hereunto, no superconductivity has been observed in isostructural materials, despite extensive investigation. Here, we report the discovery of superconductivity with a maximum $T_{\mathrm{C}}$ of $5.6 \mathrm{~K}$ in $\mathrm{V}_{3} P n \mathrm{~N}_{x}(P n=\mathrm{P}, \mathrm{As})$ phases with the anti-ppv structure, where the anion and cation positions are reversed with respect to the ppv structure. This discovery stimulates further explorations of new superconducting materials with ppv and anti-ppv structures.

S ince the discovery of high- $T_{\mathrm{C}}$ superconductivity in cuprates with the layered-perovskite (pv) structure ${ }^{1}$, extensive effort has been devoted to finding other superconducting materials. After a quarter century of investigation, many layered superconducting families have been discovered, such as a ruthenate $\mathrm{Sr}_{2} \mathrm{RuO}_{4}{ }^{2}$, boride $\mathrm{MgB}_{2}{ }^{3}$, hafnium nitride chloride ${ }^{4}$, cobaltate $\mathrm{Na}_{x} \mathrm{CoO}_{2} \cdot y \mathrm{H}_{2} \mathrm{O}^{5}$, an intercalated graphite $\mathrm{C}_{6} \mathrm{Ca}^{6}$, a chalcogenide $\mathrm{Cu}_{x} \mathrm{TiSe}_{2}{ }^{7}$, and iron-based pnictides and chalcogenides ${ }^{8,9}$. The layered characteristics of the host crystal structures are widely believed to be essential in producing superconductivity due to the anisotropic electronic structures. This provides an important reference for the design of superconducting materials and the exploration of new mechanisms for superconductivity ${ }^{1,2,4-6}$. Recently, the ppv transition of $\mathrm{MgSiO}_{3}$ was discovered using a laser-heated diamond anvil cell ${ }^{10,11}$. Consequently, this phase has received more attention because it is considered to be the main constitute of the Earth's lowermost mantle (D" layer, ca. 2700-2900 km deep) (Fig. 1a). The ppv crystal structure is comprised of alternately-stacked $\mathrm{SiO}_{6}$ octahedra and $\mathrm{Mg}$ atoms along the $b$ axis and it has typical two-dimensional characteristics. This has motivated research into the physical phenomena of this phase, including superconductivity. However, ppv- $\mathrm{MgSiO}_{3}$ is stable only under extreme conditions (120 GPa and $\left.2200^{\circ} \mathrm{C}\right)^{12}$ and is unquenchable to ambient pressure, which has restricted further research into chemical substitution and carrier doping of the structure. Therefore, there have been attempts to establish analogue materials that are stable under ambient conditions. Almost 20 ppv-type compounds have been identified to date, including the $\mathrm{MgGeO}_{3}, \mathrm{NaIrO}_{3}$, and $\mathrm{CaBO}_{3}(B=\mathrm{Ru}, \mathrm{Rh}, \mathrm{Sn}$, Ir, and $\mathrm{Pt})$ oxides, $\mathrm{Na}(\mathrm{Mg}, \mathrm{Zn}) \mathrm{F}_{3}$ fluorides and $(\mathrm{U}, \mathrm{Th}) \mathrm{MnSe}_{3}$ chalcogenides ${ }^{13-19}$. As with perovskite-type materials, many interesting physical phenomena have been observed in ppv-type materials, such as metal-insulator phase transition ${ }^{13}$ and low-dimensional magnetism ${ }^{17}$. However, no superconductivity has been reported so far for the ppv family, because most compounds with ppv structure are Mott insulators owing to the strong electron correlation effect.

In this letter, we report the observation of superconductivity in $\mathrm{V}_{3} P n \mathrm{~N}_{x}(P n=\mathrm{P}$, As). These compounds crystallize in the filled $\mathrm{Re}_{3} \mathrm{~B}$ structure with the orthorhombic $\mathrm{Cmcm}$ (\#63) space group, as depicted in Fig. $1 \mathrm{~b}^{20}$. The positions occupied by the anions and cations are opposite to those of the ppv structure. Considering also the nomenclature of the anti-pv structure ${ }^{21,22}$, we call this structure the anti-ppv structure (see crystal structure details in Section I of the Supplementary Information). The anti-ppv-type $\mathrm{V}_{3} P n \mathrm{~N}_{x}$ is composed of alternately-stacked $\mathrm{NV}_{6}$ octahedral layers and $P n \mathrm{~V}_{8}$ bicapped trigonal prisms layers along the $b$ axis, which gives rise to quasi-twodimensional electronic states. Within the $a c$-plane, $\mathrm{NV}_{6}$ octahedra are connected by edge sharing along the $a$ axis and corner sharing along the $c$ axis $^{20}$. Here, we also notice that $\mathrm{V}-\mathrm{V}$ metallic bonds play the key role in stabilizing this structure ${ }^{20}$.

\section{Results}

Figure 2a presents the electrical resistivity $(\rho)$ of $\mathrm{V}_{3} P n \mathrm{~N}_{x}(P n=\mathrm{P}, \mathrm{As})$ as a function of temperature $(T)$. The room temperature resistivities $\rho_{300 \mathrm{~K}}$, are approximately 340 and $240 \mu \Omega \mathrm{cm}$ for $\mathrm{V}_{3} \mathrm{PN}$ and $\mathrm{V}_{3} \mathrm{AsN}$, respectively. $\rho$ 
(a)

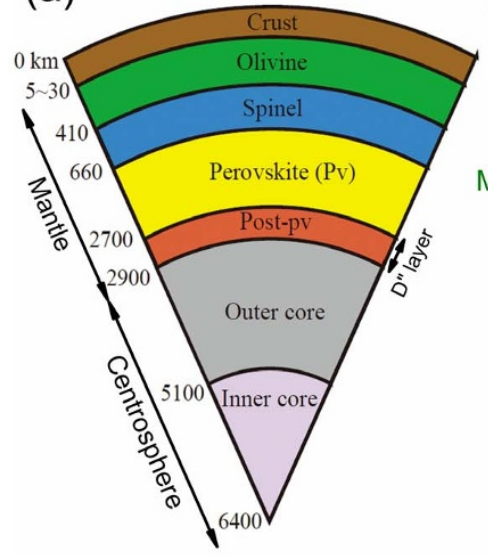

(b)

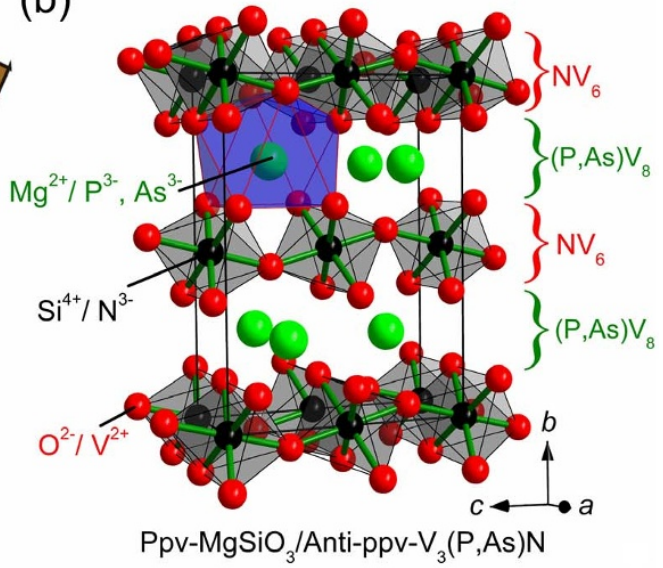

Figure 1 | Schematic illustration of Earth's interior and crystal structures of ppv- $\mathrm{MgSiO}_{3} / \mathbf{a n t i}$-ppv- $\mathrm{V}_{3}$ PnN. (a) The outermost solid shell is the crust composed of a variety of rocks. The mantle below the crust has layer structures corresponding to the structural transition induced by pressure in Mg$\mathrm{Si}-\mathrm{O}$ system. The lowermost layer is called the D" layer, where the main constitute is the ppv-type silicate. The outer and inner cores below the mantle consist of liquid and solid iron alloys, respectively. (b) Crystal structure of ppv- $\mathrm{MgSiO}_{3}$ and anti-ppv- $\mathrm{V}_{3} P n \mathrm{~N}(P n=\mathrm{P}, \mathrm{As})$ with the Cmcm (\#63) space group. Solid lines represent a unit cell. The anti-ppv structure has layer structures composed of $\mathrm{NV}_{6}$ octahedra and $P n V_{8}$ polyhedra.

decreases gradually with cooling (metallic behaviour) and drops sharply to zero at $T_{\mathrm{C}}=4.2$ and $2.6 \mathrm{~K}$ for $\mathrm{V}_{3} \mathrm{PN}$ and $\mathrm{V}_{3} \mathrm{AsN}$ (Fig. 2c), respectively, which indicates the appearance of superconductivity. The width of the transition temperature is narrow (ca. $0.3 \mathrm{~K}$ ), which implies good sample quality. Figure $2 \mathrm{~d}$ presents the magnetic susceptibility $(M / H)$ under zero-field cooling (ZFC) and field cooling (FC) conditions at $H=10$ Oe. The superconducting volume fraction estimated from $\mathrm{ZFC}$ data at $1.8 \mathrm{~K}$ are approximately 190 and $167 \%$ for $\mathrm{V}_{3} \mathrm{PN}$ and $\mathrm{V}_{3} \mathrm{AsN}$, respectively. The volume fractions exceeding $100 \%$ is attributable to the polycrystalline nature of samples. The specific heat shown in Fig. 2e has a sudden increase around $T_{\mathrm{C}}$. These results provide unambiguous evidence for the bulk superconductivity in $\mathrm{V}_{3} P n \mathrm{~N}(P n=\mathrm{P}, \mathrm{As})$.

Detailed measurements of the field-dependent resistivity and magnetization presented in Figs. 3a-f allow for better characterization of the superconducting state. The $H$-dependence of $\rho$ (Figs. $3 a-c$ ) gives the upper critical field $H_{\mathrm{C} 2}$, as shown in Fig. $3 \mathrm{~g}$. There is another weak transition above $H_{C 2}$ that originates from a small amount of the $\mathrm{VN}_{x}$ impurity phase, as shown in Fig. S1(a). Close to $T_{\mathrm{C}}, H_{\mathrm{C} 2}$ is linearly dependent on $T$ in accordance with the Werthamer-HelfandHohenberg theory ${ }^{23}$. Using the formula $H_{\mathrm{C} 2}(0)=-0.693 T_{\mathrm{C}} \mathrm{d} H_{\mathrm{C} 2} /$ $\mathrm{d} T$ provided $H_{\mathrm{C} 2}$ at the ground state; $H_{\mathrm{C} 2}(0)=34.9$ and $27.9 \mathrm{kOe}$ was obtained for $\mathrm{V}_{3} \mathrm{PN}$ and $\mathrm{V}_{3} \mathrm{AsN}$, respectively. According to the Bardeen-Copper-Schrieffer (BCS) theory ${ }^{24}$, the $H_{\mathrm{C} 2}$ value is related to the coherent length $\xi$, as $H_{\mathrm{C} 2}=\Phi_{0} / 2 \pi \xi^{2}$ (where $\Phi_{0}$ is the magnetic flux quantum). Using this formula, $\xi=9.7$ and $10.9 \mathrm{~nm}$ are obtained for $\mathrm{V}_{3} \mathrm{PN}$ and $\mathrm{V}_{3} \mathrm{AsN}$, respectively. The magnetization isotherms at $1.8 \mathrm{~K}$ exhibit typical type-II superconductor behaviour (insets of Figs. 3d, 3f). The lower critical field $H_{\mathrm{C} 1}$ was determined from the magnetic field, where the magnetization departs from the linear $H_{-}$ dependence (indicated by arrows in Figs. 3d, 3f). The temperature dependence of $H_{\mathrm{C} 1}$ is well fitted with the empirical function $H_{\mathrm{C} 1}(T)$ $=H_{\mathrm{C} 1}(0)\left[1-\alpha\left(T / T_{\mathrm{C}}\right)^{2}\right]$ (where $\alpha$ is the fitting parameter), and the lower critical fields obtained at the ground state are $H_{\mathrm{C} 1}(0)=207$ and
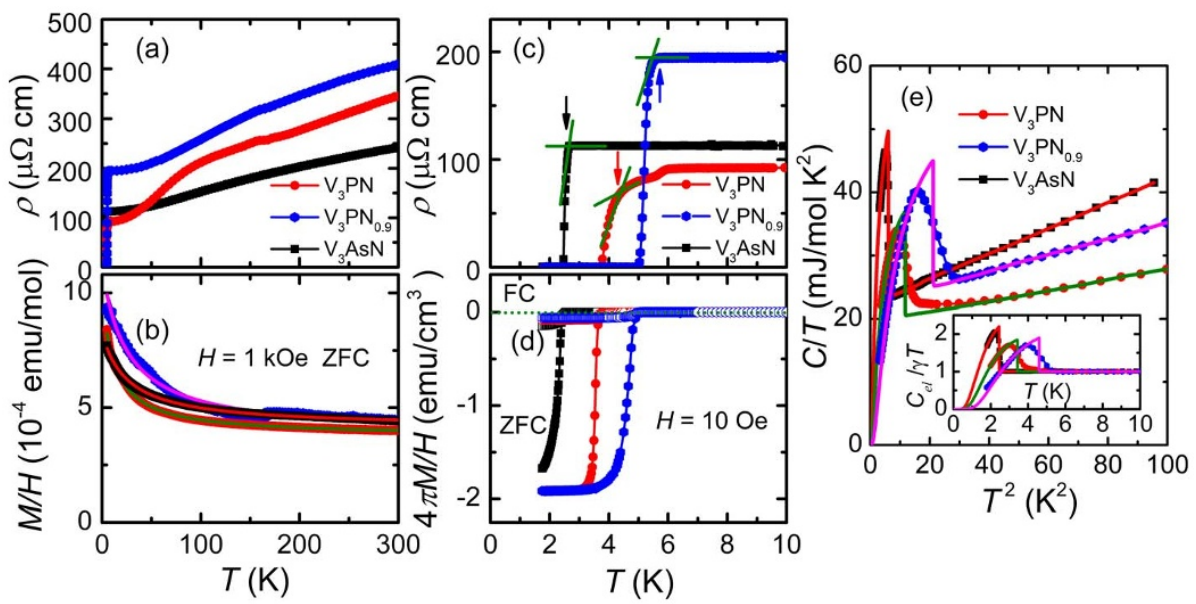

Figure $2 \mid$ Evidence for bulk superconductivity in $\mathrm{V}_{3} \operatorname{PnN}(\boldsymbol{P n}=\mathbf{P}$, As). (a) Temperature $(T)$ dependent resistivity $(\rho)$ at zero magnetic field $(H)$. (b) DC susceptibility $(M / H)$ curve under the ZFC condition at $H=1 \mathrm{kOe}$. Solid lines indicate fitting results (see text). (c) Enlargement of low-temperature resistivity data. Arrows indicate the superconducting transition temperature $\left(T_{\mathrm{C}}\right)$. (d) Low-temperature $M / H$ - $T$ curve under ZFC and FC conditions at $H$ $=10$ Oe. (e) Temperature dependence of specific heat $(C / T)$ at zero magnetic field. Solid lines indicate fitting with $C=\gamma T+\beta T^{3}$ in the normal state and the function based on the BCS model in the superconducting state. Inset shows the $C_{e l} l \gamma T_{\mathrm{C}}$ value, where $C_{e l}$ is the electron contribution of the specific heat. The solid curve is calculated from the BCS model with an isotropic gap. 


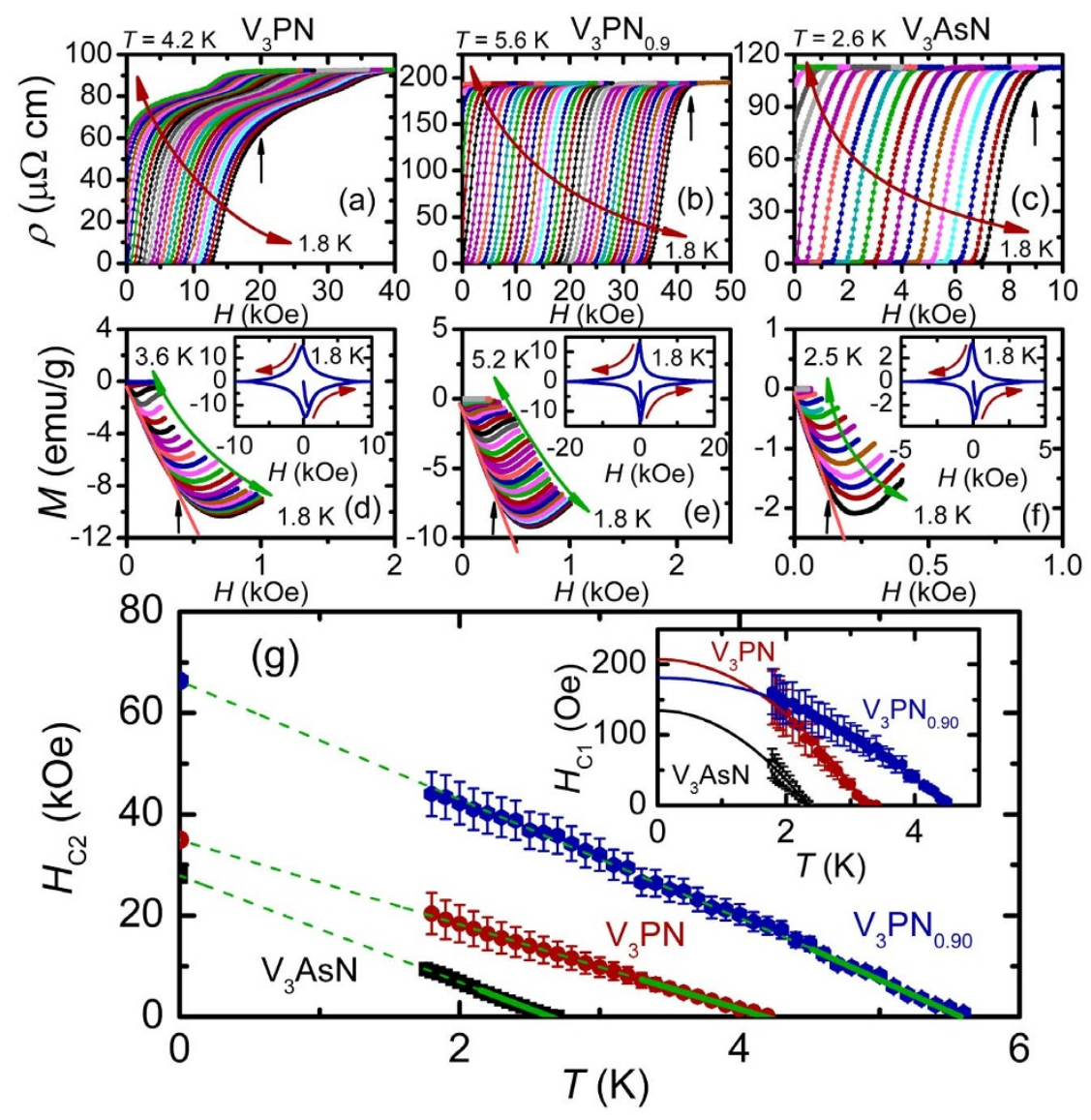

Figure $3 \mid$ Characterization of the superconductivity in $\mathrm{V}_{3} P n N(P n=P, A s)$. Magnetic field $(H)$ dependence of $(a-c)$ resistivity $(\rho)$ and $(d-f)$ magnetization $(M)$ at fixed temperatures. Arrows in $(\mathrm{a}-\mathrm{c})$ indicate the upper critical field $\left(H_{\mathrm{C} 2}\right)$ at $1.8 \mathrm{~K}$. There is another weak transition above $H_{\mathrm{C} 2}$ that originates from a small amount of the $\mathrm{VN}_{x}$ impurity phase, as shown in Fig. $\mathrm{S} 1(\mathrm{a})$. Arrows in Figs. (d-f) indicate the lower critical field ( $\left.H_{\mathrm{Cl}}\right)$, where $M$ departs from the linear $H$-dependence. Insets in $(\mathrm{d}-\mathrm{f})$ are magnetization isotherms at $1.8 \mathrm{~K}$ over a wider $H$ range. (g) Temperature ( $T$ ) dependence of the upper and lower critical fields $\left(H_{\mathrm{C} 2}\right.$ and $\left.H_{\mathrm{C} 1}\right)$. Solid lines indicate fitting results (see text).

134 Oe for $\mathrm{V}_{3} \mathrm{PN}$ and $\mathrm{V}_{3} \mathrm{AsN}$, respectively (inset of Fig. $3 \mathrm{~g}$ ). The London penetration depths $\lambda_{\mathrm{L}}$, and the Ginzburg-Landau parameters $\kappa$ are estimated from the formula $H_{\mathrm{C} 2} / H_{\mathrm{C} 1}=2 \kappa^{2} / \ln \kappa$ with $\kappa=\lambda_{\mathrm{L}} / \xi$ to be $\lambda_{\mathrm{L}}=157$ and $187 \mathrm{~nm}$ and $\kappa=16.2$ and 17.2 for $\mathrm{V}_{3} \mathrm{PN}$ and $\mathrm{V}_{3} \mathrm{AsN}$, respectively.

The appearance of superconductivity in both $P n=\mathrm{P}$ and As compounds, as well as $\mathrm{N}$-deficient systems (Fig. 4), indicate that the $\mathrm{V}-3 d$ electrons are predominantly responsible for the emergence of superconductivity. The specific heat was closely examined to reveal bosons that act as the glue for Cooper pairs. The specific heat at the normal state can be well fitted using the function $C=\gamma T+\beta$ $T^{3}$ (Fig. 2e), where the former and latter terms represent the electron and phonon contributions, respectively. We estimated $\gamma=19.5$ and $22.0 \mathrm{~mJ} / \mathrm{mol} \mathrm{K}^{2}$, and $\beta=0.083$ and $0.20 \mathrm{~mJ} / \mathrm{mol} \mathrm{K}$ for $\mathrm{V}_{3} \mathrm{PN}$ and $\mathrm{V}_{3} \mathrm{AsN}$, respectively. The $\Delta C / \gamma T_{C}$ value (inset of Fig. 2e), where $\Delta C$ is the specific heat jump at $T_{C}$, is approximately 0.86 and 1.22 for $\mathrm{V}_{3} \mathrm{PN}$ and $\mathrm{V}_{3} \mathrm{AsN}$, respectively. These values are slightly smaller than 1.43 expected for a typical BCS superconductor with a weak-coupling limit, which implies that the electron-phonon coupling is the glue for the Cooper pairs ${ }^{24,25}$. The Debye temperature was estimated to be $\theta_{\mathrm{D}}=489$ and $364 \mathrm{~K}$ for $\mathrm{V}_{3} \mathrm{PN}$ and $\mathrm{V}_{3} \mathrm{AsN}$, respectively, using the relationship $\beta=12 \pi^{4} N R / 5 \theta_{\mathrm{D}}{ }^{3}$ (where $N$ is the number of atoms in a formula unit, 5 , and $R$ is the gas constant). It is worth noting that $\theta_{\mathrm{D}}$ for $\mathrm{V}_{3} \mathrm{PN}$ is larger than that for $\mathrm{V}_{3} \mathrm{AsN}$, because materials with smaller mass exhibit harder phonons. From the McMillan formula, $T_{\mathrm{C}}=\left(\theta_{\mathrm{D}} / 1.45\right) \exp \left\{-1.04\left(1+\lambda_{p h}\right) /\left[\lambda_{p h}-\right.\right.$ $\left.\left.\mu^{*}\left(1+0.62 \lambda_{p h}\right)\right]\right\}^{26}$, together with the assumption of the Coulomb pseudopotential $\mu^{*}=0.15$, the electron-phonon coupling constants can be estimated as $\lambda_{p h}=0.55$ and 0.54 for $\mathrm{V}_{3} \mathrm{PN}$ and $\mathrm{V}_{3} \mathrm{AsN}$, respectively. These are typical values for phonon-mediated weakly coupling BCS superconductors ${ }^{24,25,27}$.

On the other hand, the importance of a strong electron correlation effect manifests itself from an analysis of the Wilson ratio $R_{\mathrm{w}}=$ $\pi^{2} k_{\mathrm{B}}^{2} \chi_{\mathrm{s}} / 3 \mu_{\mathrm{B}}^{2} \gamma$, where $k_{\mathrm{B}}$ is the Boltzmann constant, $\chi_{\mathrm{s}}$ is the spin susceptibility, and $\mu_{\mathrm{B}}$ is the Bohr magneton ${ }^{28}$. The magnetic susceptibility $(\chi=M / H)$ in the normal state exhibits weakly $T$-dependent behaviour and can be well fitted to the formula $\chi=\chi_{0}+C_{\mathrm{CW}} /(T-$ $\theta$ ) (Fig. $2 \mathrm{~b}$ ), where $\chi_{0}$ is a temperature-independent term, $C_{\mathrm{CW}}$ is the Curie-Weiss constant, and $\theta$ is the Weiss temperature. The fitting results give $\chi_{0}=4.13 \times 10^{-4}$ and $3.75 \times 10^{-4} \mathrm{emu} / \mathrm{mol}, C_{\mathrm{CW}}=1.09$ $\times 10^{-2}$ and $8.08 \times 10^{-3} \mathrm{emu} \mathrm{K} / \mathrm{mol}$, and $\theta=-26.5$ and $-13.0 \mathrm{~K}$ for $\mathrm{V}_{3} \mathrm{PN}$ and $\mathrm{V}_{3} \mathrm{AsN}$, respectively. Using the obtained $\chi_{0}$ values as $\chi_{\mathrm{s}}$, we acquire $R_{\mathrm{w}}=1.42$ and 1.45 for $\mathrm{V}_{3} \mathrm{PN}$ and $\mathrm{V}_{3} \mathrm{AsN}$, respectively. The enhancement from the Fermi liquid value of 1 indicates a moderate electron correlation effect in the present compounds. Moreover, the $\rho$ values in the normal state are in the order of $10^{-4} \Omega \mathrm{cm}$ (Figs. $2 \mathrm{a}$, $2 c$ ), which is much larger than typical $\rho$ values for conventional intermetallic compounds and indicates strong electron-electron interaction. Therefore, magnetic fluctuations originating from the strong electron correlation effect could not be excluded as the pairing glue for Cooper pairs.

Besides elucidation of the microscopic mechanism, identification of the chemical factors responsible for the appearance of superconductivity is important to further increase $T_{\mathrm{C}}$ in this new family of superconductors. Therefore, we have focused on the effects of $\mathrm{N}$ defects on the superconductivity. The $T_{\mathrm{C}}$ values determined from 

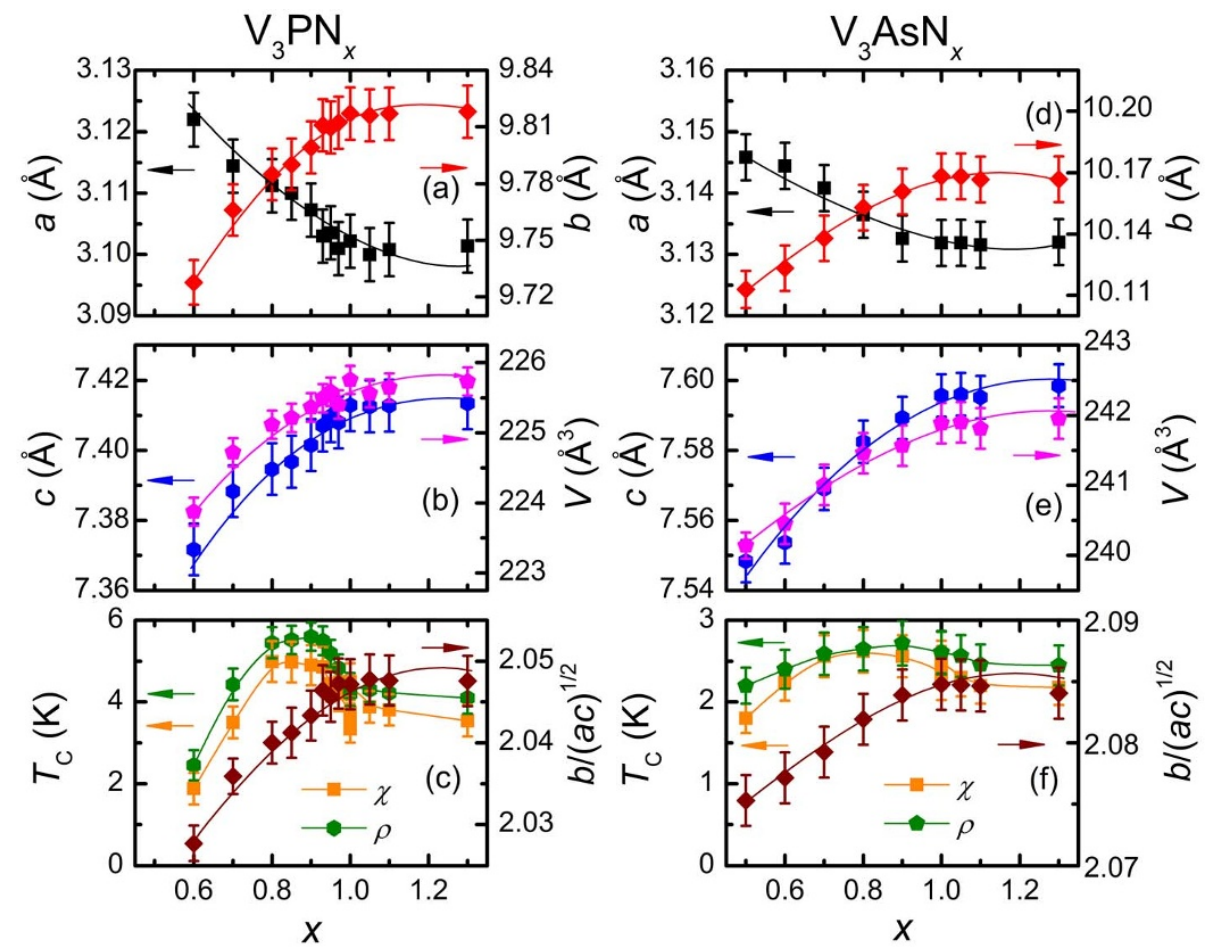

Figure $4 \mid$ Lattice parameters and superconducting transition temperature as a function of $\mathbf{N}$-content $(\boldsymbol{x})$. The values of $a, b, c, V$ and $T_{\mathrm{C}}$ were determined from resistivity $(\rho)$ and susceptibility $(\chi)$ data for $(\mathrm{a}-\mathrm{c}) \mathrm{V}_{3} \mathrm{PN}_{x}$ and $(\mathrm{d}-\mathrm{f}) \mathrm{V}_{3} \mathrm{AsN}_{x}$. The $b /[a c]^{1 / 2}$ values, which quantify the two dimensionality of the systems, are also plotted in (c) and (f).

the low-temperature resistivity and magnetization of $\mathrm{V}_{3} \mathrm{PN}_{x}(x=$ 0.6-1.3) and $\mathrm{V}_{3} \mathrm{AsN}_{x}(x=0.5-1.3)$ ( $x$ is the nominal composition, and the actual composition is expected to be less than 1) have been summarized in Figs. $4 \mathrm{c}$ and $4 \mathrm{f}$ (raw data are shown in Fig. S3 of supplementary information). For both cases, $T_{\mathrm{C}}$ is significantly enhanced with increasing $x$ in the low $x$ region, then decreases slightly after reaching a maximum at just below the stoichiometric composition $(x=1)$. The highest $T_{\mathrm{C}}$ achieved was $5.6 \mathrm{~K}$ for $\mathrm{V}_{3} \mathrm{PN}_{0.9}$, and the corresponding electronic properties are presented in Figs. $2 \mathrm{a}-\mathrm{e}, 3 \mathrm{~b}, 3 \mathrm{e}$, and $3 \mathrm{~g}$. In addition, as $x$ increases, the lattice expands along the $b$ and $c$ axes, and contracts along the $a$ axis, which results in a slight increase of the unit cell volume. Here, we note that the $b /(a c)^{1 / 2}$ value, which quantifies the two-dimensionality of the system, is well correlated with the $T_{\mathrm{C}}$ values, as shown in Figs. $4 \mathrm{c}$ and $4 \mathrm{f}$.

\section{Discussion}

On the basis of these experimental results, we now discuss the mechanism of superconductivity in $\mathrm{V}_{3} P n \mathrm{~N}$. The strong correlation between $T_{\mathrm{C}}$ and $b /(a c)^{1 / 2}$ values suggests that the quasi two dimensionality of crystal structure plays an important role in the appearance of superconductivity. This suggestion is reinforced by the higher $T_{\mathrm{C}}$ for $\mathrm{V}_{3} \mathrm{PN}$ than for $\mathrm{V}_{3} \mathrm{AsN}$. The former system has stronger intraplane coupling because the smaller atomic radius of $\mathrm{P}$ compared with that of As makes the in-plane V-V bond distances shorter, resulting in the enhanced hybridization of $3 d$ orbitals in the plane; moreover, more ionic character of V-P bonds compared with V-As bonds due to the larger electronegativity of $\mathrm{P}$ than that of As also enhances the two-dimensionality of the system. Then, how this two dimensionality favor the superconductivity? If the electron-phonon interaction mediates Cooper pairs as suggested by electronic properties, the $T_{\mathrm{C}}$ value is considered to be enhanced by the larger density of states at the Fermi energy in low dimensional systems. Another possibility is that the electron correlation effect pronounced in the low dimensional crystal structure due to the smaller kinetic energy of electrons stabilizes the superconducting states. Generally, $T_{\mathrm{C}}$ is known to be very sensitive to various factors in reported superconductors. Therefore, further detailed studies are required to identify the mechanism of superconductivity, especially on hybridization of the V-3d and $\mathrm{N}-2 p$ bands, the direct-overlapping of $\mathrm{V}-3 d$ orbitals across two $\mathrm{V}$ atoms, and the change in carrier density introduced by $\mathrm{N}$-defects.

To summarize, we have discovered superconductivity with maximum $T_{\mathrm{C}}$ at 5.6 and $2.6 \mathrm{~K}$ for $\mathrm{V}_{3} \mathrm{PN}_{x}$ and $\mathrm{V}_{3} \mathrm{AsN}_{x}$ with the anti ppv structure. Two-dimensionality is the key for the appearance of superconductivity; however, to elucidate the microscopic mechanism of superconductivity, further experimental and theoretical studies are required. These findings should stimulate future experimental and theoretical research on ppv-type materials to explore advanced functionalities.

\section{Methods}

An optimized synthesis method ${ }^{20}$ was employed. Powders of elemental vanadium (99.999\%), vanadium nitride (99.9\%), and phosphorus (99.99\%) or arsenic (99.9\%) were mixed in a stoichiometric ratio, pressed into pellets in a nitrogen-filled glove box, and then sealed in a quartz tube under 0.3 atm of argon gas. The quartz tube was slowly heated to $673 \mathrm{~K}$, held for $24 \mathrm{~h}$ to avoid rapid volatilization of the phosphorus or arsenic, then heated to $1273 \mathrm{~K}$ for $12 \mathrm{~h}$ and held for $120 \mathrm{~h}$. After quenching the tubes to room temperature, the product was pulverized and pressed into pellets. The pellets were annealed inside a quartz tube at $1273 \mathrm{~K}$ for $48 \mathrm{~h}$. To remove oxide impurities during this procedure, the $\mathrm{V}_{3} \mathrm{PN}_{x}$ and $\mathrm{V}_{3} \mathrm{AsN}_{x}$ pellets were wrapped with molybdenum and tantalum foil, respectively, inside the quartz tube. The as-synthesized samples were dark grey coloured with a metallic luster and were stable in air. The samples were characterized using powder X-ray diffraction (Rigaku, Smartlab) with $\mathrm{Cu} \mathrm{K} \alpha$ radiation.

The detailed structural parameters were obtained by Rietveld refinement using Rietica software ${ }^{29}$. Details of the analysis are presented in section I of the Supplementary Information. Magnetic, electrical, and heat capacity measurements were performed using a commercial apparatus (Quantum Design) from 1.8 to $300 \mathrm{~K}$. DC resistivity measurements were performed using the four-probe method with gold paste as electrodes.

1. Bednorz, J. G. \& Müller, K. A. Possible high $T_{\mathrm{C}}$ superconductivity in the Ba-LaCu-O system. Z. Phys. B 64, 189-193 (1986). 
2. Maeno, Y. et al. Superconductivity in a layered perovskite without copper. Nature 372, 532-534 (1994).

3. Nagamatsu, J. et al. Superconductivity at $39 \mathrm{~K}$ in magnesium diboride. Nature 410, 63-64 (2001).

4. Yamanaka, S. et al. Superconductivity at $25.5 \mathrm{~K}$ in electron-doped layered hafnium nitride. Nature 392, 580-582 (2001).

5. Takada, K. et al. Superconductivity in two-dimensional $\mathrm{CoO}_{2}$ layers. Nature 422, 53-55 (2003).

6. Thomas, E. W. et al. Superconductivity in the intercalated graphite compounds $\mathrm{C}_{6} \mathrm{Yb}$ and $\mathrm{C}_{6} \mathrm{Ca}$. Nature Phys. 1, 39-41 (2005).

7. Morosan, E. et al. Superconductivity in $\mathrm{Cu}_{x} \mathrm{TiSe}_{2}$. Nature Phys. 2, 544-550 (2006).

8. Kamihara, Y. et al. Iron-based layered superconductor $\mathrm{La}\left[\mathrm{O}_{1-x} \mathrm{~F}_{x}\right] \mathrm{FeAs}(x=0.05$ 0.12 ) with $T_{C}=26$ K. J. Am. Chem. Soc. 130, 3296-3297 (2008).

9. Chen, X. H. et al. Superconductivity at $43 \mathrm{~K}$ in $\mathrm{SmFeAsO}_{1-x} \mathrm{~F}_{x}$. Nature 435 , 761-762 (2008).

10. Murakami, M. et al. Post-perovskite phase transition in $\mathrm{MgSiO}_{3}$. Science 304, 855-858 (2004).

11. Oganov, A. R. \& Ono, S. Theoretical and experimental evidence for a postperovskite phase of $\mathrm{MgSiO}_{3}$ in earth's D" layer. Nature 430, 445-448 (2004).

12. Belonoshko, A. B. et al. High-pressure melting of $\mathrm{MgSiO}_{3}$. Phys. Rev. Lett. 94, 195701 (2005).

13. Ohgushi, K. et al. Metal-insulator transition in $\mathrm{Ca}_{1-x} \mathrm{Na}_{x} \mathrm{IrO}_{3}$ with post-perovskite structure. Phys. Rev. B. 74, 241104(R) (2006).

14. Yamaura, K. et al. Synthesis and magnetic and charge-transport properties of the correlated 4d post-perovskite $\mathrm{CaRhO}_{3}$. J. Am. Chem. Soc. 131, 2722-2726 (2009).

15. Cheng, J.-G. et al. Stress-induced perovskite to post-perovskite in $\mathrm{CaIrO}_{3}$ at room temperature. Phys. Rev. B. 82, 132103 (2010).

16. Bremholm, M. et al. $\mathrm{NaIrO}_{3}$-A pentavalent post-perovskite. J. Solid State Chem. 184, 601-607 (2011)

17. Shirako, Y. et al. Integer spin-chain antiferromagnetism of the $4 d$-oxide $\mathrm{CaRuO}_{3}$ with post-perovskite structure. Phys. Rev. B. 83, 174411 (2011).

18. Yakovlev, S. et al. High-pressure structural behavior and equation of state of $\mathrm{NaZnF}_{3}$. J. Solid State Chem. 182, 1545-1549 (2009).

19. Ijjaali, I. et al. Syntheses and characterization of the actinide manganese selenides $\mathrm{ThMnSe}_{3}$ and $\mathrm{UMnSe}_{3}$. J. Solid State Chem. 177, 257-261 (2004).

20. Boller, H. \& Nowotny, H. Komplexcarbide und-nitride mitaufgefülltem $\operatorname{Re}_{3} B$ type. Monatsh. Chem. 99, 721-725 (1968).

21. He, T. et al. Superconductivity in the non-oxide perovskite $\mathrm{MgCNi}_{3}$. Nature 411 , 54-56 (2001).

22. Takayama, T. et al. Strong coupling superconductivity at $8.4 \mathrm{~K}$ in an antiperovskite phosphide $\mathrm{SrPt}_{3}$ P. Phys. Rev. Lett. 108, 237001 (2012).
23. Werthamer, N. R. et al. Temperature and purity dependence of the superconducting critical field, $H_{\mathrm{C} 2}$. III. Electron spin and spin-orbit effects. Phys. Rev. 147, 295-302 (1966).

24. Bardeen, J., Cooper, L. N. \& Schrieffer, J. R. Theory of superconductivity. Phys. Rev. 108, 1175-1204 (1957)

25. Kresin, V. Z. On the critical temperature for any strength of the electron-phonon coupling. Phys. Lett. A 122, 434-438 (1987).

26. McMillan, W. L. Transition temperature of strong-coupled superconductors. Phys. Rev. 167, 331-344 (1968).

27. Kresin, V. Z. \& Parchomenko, V. Thermodynamic properties of strong-coupling superconductors. Sov. Phys. Solid State 16, 2180-2184 (1975).

28. Wilson, K. The renormalization group: Critical phenomena and the Kondo problem. Rev. Mod. Phys. 47, 773-840 (1975).

29. Rietveld, H. M. A profile refinement method for nuclear and magnetic structures. J. Appl. Cryst. 2, 65-71 (1969).

\section{Acknowledgments}

The authors acknowledge discussions with Prof. Y. Ueda, Dr. Y. Hirata, Dr. Y. Q. Zhang, and Dr. F. Du. We also thank M. Isobe and T. Yamauchi for their technical supports. This work was supported by the Grant Program of the Sumitomo Foundation, and the Grant Program of the Murata Science Foundation.

\section{Author contributions}

B.W. prepared the samples and carried out the experiments. The authors equally contributed to analysing the results. B.W. wrote the paper with assistance from K.O. K.O. directed the research.

\section{Additional information}

Supplementary information accompanies this paper at http://www.nature.com/ scientificreports

Competing financial interests: The authors declare no competing financial interests.

How to cite this article: Wang, B. \& Ohgushi, K. Superconductivity in anti-post-perovskite vanadium compounds. Sci. Rep. 3, 3381; DOI:10.1038/srep03381 (2013).

(c) (1) $\Theta$ This work is licensed under a Creative Commons Attribution-

By NG No NonCommercial-NoDerivs 3.0 Unported license. To view a copy of this license, visit http://creativecommons.org/licenses/by-nc-nd/3.0 